\title{
Long-term differences in urinary, bowel and sexual function among men treated with surgery versus radiation for prostate cancer
}

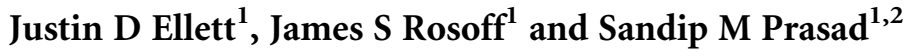 \\ Asian Journal of Andrology (2013) 15, 443-444; doi:10.1038/aja.2013.39; published online 13 May 2013
}

\begin{abstract}
urgery and radiation have both been shown to increase the long-term disease-specific survival rate for men with clinically localized prostate cancer. Although both modalities have demonstrated favorable effects on cancer control, questions regarding quality of life (QoL) and functional outcomes remain incompletely answered.
\end{abstract}

To date, no randomized prospective trials have been performed comparing the two treatment modalities and so indirect comparisons of long-term functional outcomes have served as a substitute to aid in patient counseling and decision-making. As there is a paucity of long-term data comparing functional outcomes after radical prostatectomy and external beam radiation therapy, a recent article by Resnick et al., ${ }^{1}$ has attempted to provide additional information about this topic in terms of continence, erectile function and bowel function.

Utilizing the Prostate Cancer Outcomes Study cohort, a population-based cohort of men diagnosed with prostate cancer in the prostate-specific antigen (PSA) era, the authors compared rates of urinary incontinence, erectile dysfunction, and bowel urgency at 2, 5 and 15 years after primary therapy. They showed that men undergoing prostatectomy had higher rates of incontinence and erectile dysfunction at 2 and 5 years, but these rates were similar to those in the radiotherapy group at 15 years. More specifically, men were approximately five times more likely to have urinary continence issues if they underwent prostatectomy versus radiotherapy and almost three and

${ }^{1}$ Department of Urology, Medical University of South Carolina, Charleston, SC 29425, USA and ${ }^{2}$ Section of Urology, Ralph $H$. Johnson VA Medical Center, Charleston, SC 29401, USA

Correspondence: Dr SM Prasad (prasads@musc.edu) a half times more likely to develop erectile dysfunction in the short- to intermediate-term following primary treatment. As expected, rates of bowel urgency were higher in the radiotherapy group at 2 and 5 years, but not significantly different from the surgery group at 15 years.

In addition, the authors note that the rate of incontinence and erectile function progressively worsened over time, regardless of primary treatment modality. At 15 years of follow-up, the prevalence of erectile dysfunction was approximately $87 \%$ in the prostatectomy group, and $94 \%$ in the radiotherapy group, a non-significant difference. Interestingly, only approximately $40 \%$ of patients in either group reported being bothered by this. Without an appropriate control group, it is hard to distinguish the relative contribution of intervention or age to the overall decline in sexual function. Short-term studies have shown that men undergoing prostatectomy have larger declines in sexual and urinary function than age-matched controls, ${ }^{2}$ but no such untreated control cohort was present in this study.

The effects on sexual, urinary and bowel function are critical issues to address when counseling patients regarding prostate cancer treatment. Rather than looking at specific points in time, the overall decrement in each QoL domain can be evaluated as the area under the curve for each treatment type. Therefore, while values generally are similar at 15 years, men have a cumulative difference in preserved erectile and urinary function over that period that can be compared by area under the curve measurement. A calculation of the relative decrement in each domain over time would be valuable for patient counseling, but these are not provided by the authors. In addition, the generalizability of the authors' findings may be limited by the dramatic refinement of treatment modalities since study enrollment in the mid-1990s. Robot-assisted laparoscopic radical prostatectomy is now the primary surgical therapy for prostate cancer, with a much smaller proportion of prostatectomies being performed at low-volume centers and those not offering robotic surgery. ${ }^{3}$ As the shift to higher volume surgeons has progressed, it is reasonable to consider that the improvements in lengths of stay and short-term outcomes after robotic surgery could be extrapolated to the continence and sexual function domains. ${ }^{4}$ Additionally, the advent of image modulation in radiotherapy has reduced late toxicity rates and, in the current era, may lower the reported rates of erectile dysfunction, urinary incontinence and bowel dysfunction reported in this study. ${ }^{5}$

Any assessment of QoL following prostate cancer treatment merits discussion of the overdiagnosis and overtreatment of clinically insignificant cancers. Less than $10 \%$ of patients in either cohort had a Gleason score $\geqslant 8$ and less than a third of patients had a PSA level $>10 \mathrm{ng} \mathrm{ml}^{-1}$. There has been an increasing view that some Gleason 6 prostate cancers do not have metastatic potential. As such, active surveillance in men with low-risk disease is an appropriate choice and minimizes treatment-specific issues with the QoL parameters considered in this study. ${ }^{6}$ Selection of only those men who have a high likelihood of benefiting from treatment may have the most significant effect in reducing treatmentrelated sexual, bladder and bowel dysfunction. ${ }^{7}$ The Prostate Cancer Research International Active Surveillance Project is the largest observational prospective study evaluating active surveillance as an alternative to radical treatment for low-risk prostate cancer. Their data show that surveillance is a feasible strategy that does not compromise cancer cure. While 
neg

Research Highlight

444

follow-up is short, the authors utilized PSA kinetics as a monitoring strategy to transition patients to treatment if they had progression of risk. ${ }^{8}$ Other groups have incorporated the use of multiparametric MRI and serum biomarkers to improve identification of patients at higher risk of prostate cancer death. ${ }^{9,10}$

There are several important limitations of this study. Men were asked to recall their baseline urinary, sexual and bowel function prior to therapy at a time point 6 months after they had undergone treatment, a criticism of all Prostate Cancer Outcomes Study-based studies. While the data suggest that estimates are unlikely to change over time, we do not know if men who have undergone surgery with worse immediate continence and erectile function are more likely to experience recall bias than men undergoing radiation, who are less likely to have any significant BoL issues at that time. While the study groups were balanced with regard to disease severity, the results were not stratified by these parameters. In addition, one of the major findings of the study was that all men showed a decrease in sexual function and continence over time. This finding highlights the imporrance of including a non-treated control group. Finally, there was significant loss to follow-up and incomplete data sets, as would be expected with a study of this length and size. The authors use complex modeling to predict responses for patients, but the nonrandom loss of patients $(60 \%$ response rate among living patients at 15 years) is difficult to control for. Furthermore, both death and loss to follow-up may have decreased the sample size sufficiently by 15 years to increase the likelihood of committing a type II error.

This study provides important insight into the long-term outcomes in erectile function, contnonce and bowel function after radical prostatectomy and radiotherapy for prostate cancer. As the largest study with the longest follow-up in the literature to date, this report should be incorporated into the discussion of post-treatment BoL for men with prostate cancer.

1 Resnick MJ, Koyama T, Fan KH, Albertsen PC, Goodman $\mathrm{M}$ et al. Long-term functional outcomes after treatment for localized prostate cancer. N Eng J Med 2013; 368: 436-45.

2 Hoffman RM, Gilliland FD, Penson DF, Stone SN, Hunt WC et al. Cross-sectional and longitudinal comparisons of health-related quality of life between patients with prostate carcinoma and matched controls. Cancer 2004; 101: 2011-9.

3 Stitzenberg KB, Wong YN, Nielsen ME, Egleston BL, Uzzo RG. Trends in radical prostatectomy: centralization, robotics, and access to urologic cancer care. Cancer 2012; 118: 54-62.

$4 \mathrm{Hu}$ JC, Gold KF, Pashos CL, Meta SS, Litwin MS. Role of surgeon volume in radical prostatectomy outcomes. J Chin Oncol 2003; 21: 401-5.

5 Martin JM, Bayley A, Bristow R, Chung P, Gospodarowicz $\mathrm{M}$ et al. Image guided dose escalated prostate radiotherapy: still room to improve. Radial Oncol 2009; 4: 50.

6 Klotz L. Active surveillance for prostate cancer: overview and update. Cur Treat Options Oncol 2013; 14: 97-108.

7 Sandhi GS, Andriole GL. Overdiagnosis of prostate cancer. J Natl Cancer Inst Monogr 2012; 2012 : 146-51.

8 Bul M, Zhu X, Valdagni R, Pickles T, Kakehi Y et al. Active surveillance for low-risk prostate cancer worldwide: the PRIAS Study. Eur Ural 2013; 63: 597-603.

9 Lions ML, him CR, Yang H, Chaos S, Bong CW et al. Blood-based biomarkers of aggressive prostate cancer. PLOS ONE 2012; 7: e45802.

10 Yerram NK, Volkin D, Turkey B, Nix J, Hoang AN et al. Low suspicion lesions on multiparametric magnetic resonance imaging predict for the absence of high-risk prostate cancer. BJU Int 2012; 110: E783-8.

Asian Journal of Andrology 